\title{
Wie weiter mit der dezentralen Besiedlung in der Schweiz? Abschätzung von Entwicklungsperspektiven auf Gemeindeebene
}

\section{Birgit Kopainsky, Peter Rieder, Zürich}

\section{Problemanalyse}

Strukturschwache ländliche Regionen stehen vor der Herausforderung, sich den veränderten wirtschaftlichen und sozialen Rahmenbedingungen anzupassen. Bevölkerungsrückgang, demographischer Wandel und eine Verringerung der Wirtschaftsbasis schränken die Entwicklungsperspektiven jedoch stark ein. Deshalb wird das politische Ziel der Erhaltung der dezentralen Besiedlung gelegentlich in Frage gestellt. Gleichzeitig befindet sich die schweizerische Regionalpolitik in einer Phase der grundsätzlichen Neuorientierung (EIDGENÖsSISCHES VOLKSWIRTSCHAFTSDEPARTEMENT, EVD 2004). Der Umgang mit peripheren und schlecht erreichbaren Gebieten stellt die Neue Regionalpolitik vor besondere Herausforderungen. Daraus entsteht ein Bedarf an Entscheidungsgrundlagen, die auf einer integrierten Sicht der Prozesse und Akteure im Bereich der ländlichen Entwicklung beruhen.

Der vorliegende Artikel bezieht sich auf die lokale Ebene der Beschäftigungs- und Bevölkerungsentwicklung in gefährdeten Gemeinden der Schweiz. Nach Buchur et al. (2005) wird eine Gemeinde dann als gefährdet bezeichnet, wenn ihre Bevölkerung einerseits stark abnimmt und andererseits keine Stabilisierung auf tieferem Niveau erwartet werden kann. Im Jahr 2000 konnten ca. 8\% der Gemeinden in der Schweiz nach dieser Definition als gefährdet betrachtet werden. Im Zentrum des Artikels steht die Frage der Zukunftsperspektiven dieser Gemeinden. Speziell interessiert, wie sich Beschäftigung und Bevölkerung in gefährdeten Gemeinden im Zuge wirtschaftlicher und politischer Veränderungen entwickeln und wie diese Entwicklung durch politische Massnahmen und lokale Initiativen beeinflusst werden kann. Zur Beantwortung dieser Frage entwickeln wir ein dynamisches Simulationsmodell, dessen Grundstruktur von bestehenden Theorien aus der Regionalwirtschaft und der ländlichen Entwicklungsforschung abgeleitet wird.

Die Probleme ländlicher Entwicklung und die Möglichkeiten einzelner regionalpolitischer Instrumente sind weitgehend bekannt (vgl. beispielsweise ABEGG 2005; BUSER 2005; FLURY 2002; KÜPFER 2000; MÜHLINGHAUS 2002, 2002a; SCHMID 2004; SCHMID et al. 2004; STALDER 2001). Der Beitrag dieses Artikels besteht darin, dass er die komplexen Interaktionen zwischen den
Bestimmungsfaktoren der Beschäftigungs- und Bevölkerungsentwicklung in gefährdeten Gemeinden in einem dynamischen Simulationsmodell abbildet und die resultierende Dynamik ausleuchtet.

Dabei geht es darum, die grundsätzlichen Entwicklungsmuster in gefährdeten Gemeinden zu erfassen und zu untersuchen, wie Beschäftigung und Bevölkerung auf Veränderungen in ihrem ökonomischen, sozialen und politischen Umfeld reagieren. Daraus sollen Hinweise auf die grundlegende Ausrichtung von Entwicklungsstrategien und Politikmassnahmen gewonnen werden. Es ist hingegen nicht Ziel der Analyse, einzelne Gemeinden detailliert abzubilden und daraus ein Handlungsprogramm für die entsprechenden Gemeinden abzuleiten.

In Kapitel 2 werden die Grundlagen der dynamischen Simulation beschrieben. Kapitel 3 umfasst die Synthese bestehender theoretischer Ansätze im Hinblick auf Entwicklungsperspektiven gefährdeter Gemeinden. Auf der Basis der dynamischen Simulation wird in Kapitel 4 das Verhalten von Schlüsselindikatoren der dezentralen Besiedlung im zeitlichen Verlauf aufgezeigt. Ebenso wird die Wirkung verschiedener externer Einflüsse und ausgewählter Entwicklungsstrategien auf die dezentrale Besiedlung untersucht. Aus der qualitativen und quantitativen Analyse der Bestimmungsfaktoren und Prozesse ergibt sich eine Reihe von Schlussfolgerungen für die zukünftige Entwicklung der dezentralen Besiedlung (Kapitel 5).

\section{Grundlagen der dynamischen Simulation}

Dynamische Simulationsmodelle beschreiben Ursache-Wirkungsbeziehungen und bestehen aus zwei grundsätzlichen Komponenten. Einerseits bilden sie die physische Welt ab, die für das jeweilige Problem relevant ist. Andererseits muss ein Simulationsmodell das Verhalten der Akteure im System erfassen, also wie Akteure auf verschiedene Situationen reagieren und Entscheidungen treffen. Die Verhaltenskomponente ist im Modell in Form von Entscheidungsregeln abgebildet (STERMAN 1988).

Ausserdem ermöglichen Simulationsmodelle, Rückkopplungseffekte, Nichtlinearität und Verzögerungen $\mathrm{zu}$ untersuchen. Ein Rückkopplungseffekt besteht dann, wenn sich die von einer Handlung verursachte Information durch das System bewegt, um schliesslich 
wieder am Ausgangspunkt anzulangen. Das dynamische Verhalten entsteht aus der zeitlichen Sequenz von Information, die eine Handlung bewirkt, welche ihrerseits Konsequenzen hat und weitere Handlungen veranlasst (RICHARDSON 1991).

Ein Rückkopplungseffekt kann positiv oder negativ sein. Er ist negativ, wenn die Veränderung einer Komponente dazu führt, dass die anderen Komponenten dieser Veränderung entgegenwirken. Negative Rückkopplungseffekte bewirken beispielsweise, dass sich Angebot und Nachfrage, in Abwesenheit anderer Einflüsse, auf ein Gleichgewicht hinzubewegen. Das Wort negativ beinhaltet dabei keine Bewertung. Vielmehr deutet es darauf hin, dass anfängliche Veränderungen kompensiert werden. Ein positiver Rückkopplungseffekt hingegen führt dazu, dass anfängliche Veränderungen im System verstärkt werden. Das System bewegt sich damit von einem Gleichgewicht weg (RUTH \& HaNNON 1997). Die verstärkende Wirkung zwischen Beschäftigung und Bevölkerung stellt beispielsweise einen positiven Rückkopplungseffekt dar. Im Fall der Fragestellung dieses Beitrages führt dieser Prozess zu einer gegenseitigen Erosion der Bevölkerungsbasis und der Beschäftigungsmöglichkeiten vor Ort.

Auf der Grundlage von Ausgangs- und Parameterwerten beschreiben dynamische Simulationsmodelle Veränderungen von Schlüsselindikatoren im zeitlichen Verlauf. Die Modelle erlauben die Abbildung von Veränderungsprozessen und die Abschätzung der kurz- und langfristigen Auswirkungen von Entwicklungsstrategien. Mathematisch besteht die Struktur eines formalen dynamischen Simulationsmodells aus einem System miteinander verbundener Differentialgleichungen erster Ordnung. Ausführliche Erläuterungen zur Rückkopplungseffekt-basierten dynamischen Simulation finden sich beispielsweise in Forrester (1961), Richardson \& Pugh (1999), Coyle (1996) und Sterman (2000).

\section{Prozesse der Beschäftigungs- und Bevölkerungs- entwicklung}

Die Variablen im Simulationsmodell und ihre Beziehungen untereinander werden aus Theorien der Regionalökonomie und der ländlichen Entwicklungsforschung abgeleitet. In der Diskussion um wirtschaftliche Entwicklung wird in der ländlichen Entwicklungsforschung die Organisation des Wirtschaftsprozesses im ländlichen Raum untersucht. Die Regionalökonomie beschäftigt sich vor allem mit den Wechselwirkungen zwischen den Produktionsfaktoren Arbeit und Kapital, die je nach Anwendung um weitere Faktoren wie lokales Milieu und Innovation ergänzt werden. Aus der Vielzahl bestehender Theorien identifiziert TERLuIN
(2003) diejenigen, die in der Lage sind, die tatsächliche Beschäftigungsentwicklung im ländlichen Raum zu erklären. Um die Rückkoppelungsprozesse in diesen Theorien explizit zu machen, wurden ausserdem Theorien des kollektiven Handelns und der Innovationsimplementierung berücksichtigt (KOPAINSKY 2005).

Die aus den Theorien abgeleitete Modellstruktur besteht aus mehreren Modulen, die in Abbildung 1 dargestellt sind. Das Modell beruht auf der sich verstärkenden Wirkung (positiver Rückkopplungseffekt) zwischen den Modulen Bevölkerung, Faktormarkt und Produktmarkt.

Wenn sich die Güterproduktion auf dem Produktmarkt erhöht, werden langfristig zusätzliche Arbeitskräfte auf dem Faktormarkt eingesetzt. Die zusätzliche Arbeitsnachfrage muss mit Arbeitskräften aus anderen Regionen oder Wirtschaftssektoren gedeckt werden. Dies kann durch Einpendler oder Zuwanderer geschehen. Der Entscheid zur Zuwanderung hängt stark von der Attraktivität der Gemeinde als Lebens-, Wirtschafts- und Wohnraum ab und wird massgeblich durch die Breite des Angebotes an Gütern und Dienstleistungen beeinflusst. Eine allfällige Zuwanderung von Arbeitskräften erhöht die Bevölkerungszahl im Modul Bevölkerung. Dadurch wiederum steigt der lokale Konsum, und die Güterproduktion auf dem Produktmarkt wird weiter erhöht.

Die Güterproduktion hängt allerdings zu einem grossen Teil von der Produktion für die externe Nachfrage ab. Die externe Nachfrage umfasst in unserem Modell die Exportnachfrage nach lokalen Gütern, die touristische Nachfrage und die öffentliche Nachfrage (z.B. Direktzahlungen an die Landwirtschaft, Finanzausgleich). Die sich verstärkende Wirkung zwischen den Modulen Produktmarkt, Faktormarkt und Bevölkerung wird also in entscheidender Weise durch die Produktion für die externe Nachfrage im Modul externe Akteure beeinflusst. Die Erschliessung der externen Nachfrage kann in zweifacher Hinsicht gefördert werden.

Zum einen besteht die Möglichkeit, im Rahmen der Infrastrukturpolitik die Erreichbarkeit von Exportmärkten zu erhöhen (Reduktion von Transportkosten) oder die Wettbewerbsfähigkeit der lokalen Produktion im Vergleich zu anderen Regionen zu steigern (Produktivitätssteigerung im Zuge des Technologietransfers). Bei der Steigerung der Faktorproduktivität muss allerdings berücksichtigt werden, dass diese nur in geringem Ausmass beschäftigungswirksam ist. Sie stellt vielmehr eine Voraussetzung dafür dar, dass die lokale Produktion wettbewerbsfähig wird oder bleibt.

Zum anderen kann die externe Nachfrage auch über lokale Entwicklungsinitiativen erschlossen werden 


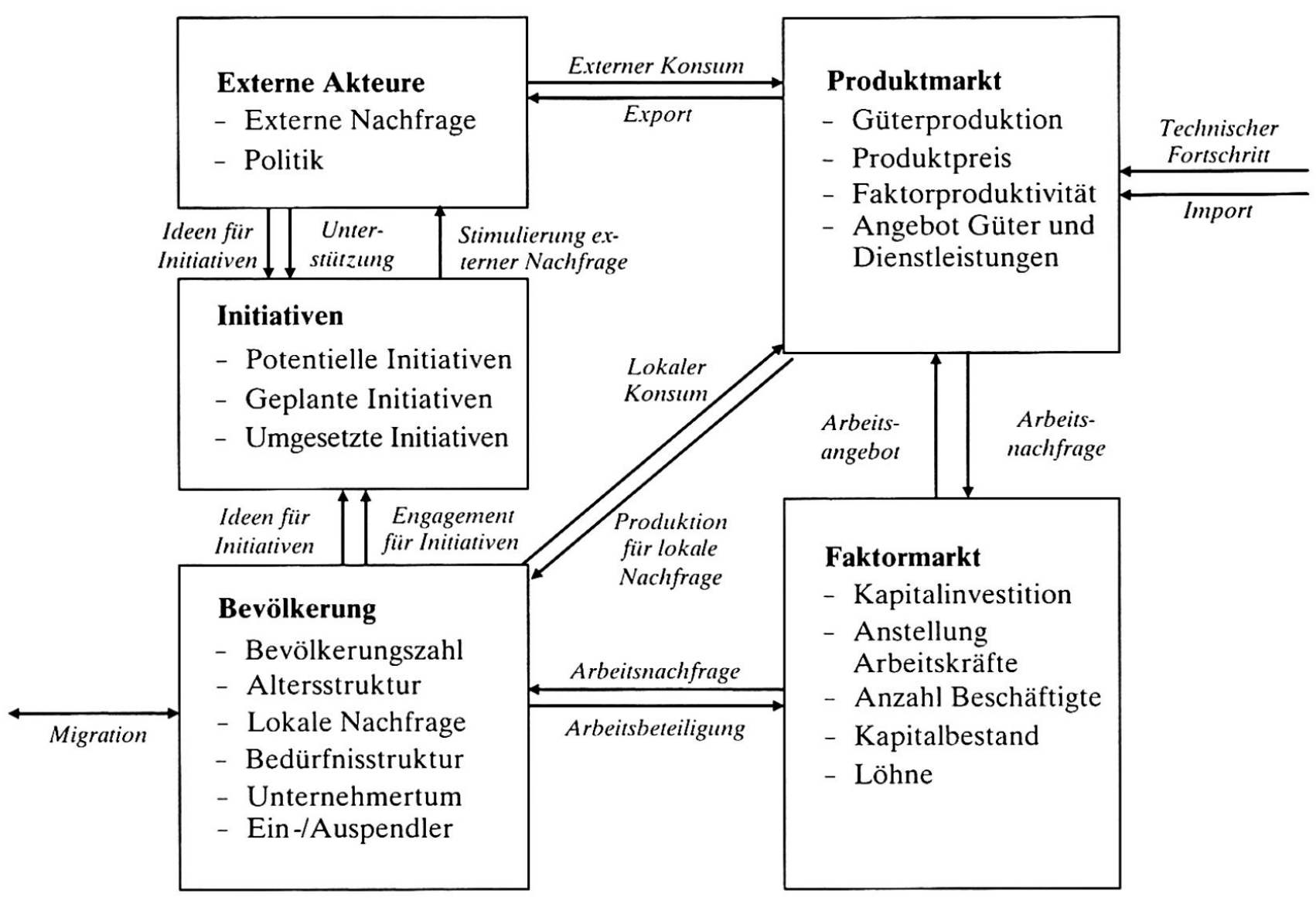

Abbildung 1: Aggregierte Struktur des dynamischen Simulationsmodells

Aggregated structure of the dynamic simulation model Structure agrégée du modèle dynamique de simulation Quelle: KopaINSKY 2005

(Modul-Initiativen). In Anlehnung an Mühlinghaus $(2002,2002 \mathrm{a})$ bezeichnen lokale Initiativen Aktivitäten von Einzelpersonen, Gruppierungen und Behörden, welche innovativen Charakter aufweisen und zur Verbesserung der lokalen Lebens- und Wirtschaftssituation beitragen. Für die Entwicklung interessant sind Initiativen, welche die Exporte von lokalen Gütern und Dienstleistungen erhöhen. Der Erfolg von lokalen Entwicklungsinitiativen hängt stark vom Engagement und der Unterstützung der beteiligten Akteure ab. Dabei stellt die Wechselwirkung zwischen Engagement und Erfolg einen positiven Rückkopplungseffekt dar. Erfolgreiche Initiativen erschliessen die externe Nachfrage, wodurch der positive Rückkopplungseffekt zwischen Produktmarkt, Faktormarkt und Bevölkerung entweder verstärkt oder überhaupt erst aktiviert wird.

Aus dem Zusammenspiel zwischen diesen Kräften, die in Abbildung 1 dargestellt sind, ergibt sich das Ausmass der Migration auf Gemeindeebene.

\section{Daten und Quantifizierung}

Die Quantifizierung des Simulationsmodells beruht im Bereich des ökonomischen Grundmodells auf der umfassenden regionalökonomischen Literatur (für eine Zusammenfassung vgl. z.B. ARMSTRONG \& TAYLOR 2000). Diese wird ergänzt durch analytische Arbeiten, die in engem Bezug zur vorliegenden Arbeit stehen. Input-Output-Analysen für gefährdete Regionen in der Schweiz (BUSER 2005) sind beispielsweise eine wichtige Grundlage für die Erfassung der ökonomischen Beziehungen und für die Schätzung der ökonomischen Parameter.

Die weiteren Modellbereiche beruhen entweder auf statistischen Daten (landwirtschaftliche Strukturerhebung, Betriebszählung, Produktions- und Wertschöpfungsstatistik, Sterbetafeln, Volkszählung), auf der Literatur zu Fallstudien (Mühlinghaus 2002, 2002a; Shucksmith 2000) oder auf der Literatur zur Innovationsimplementierung (ABRAHAMSON \& ROSENKOPF 1997; REPENNING 2002). 

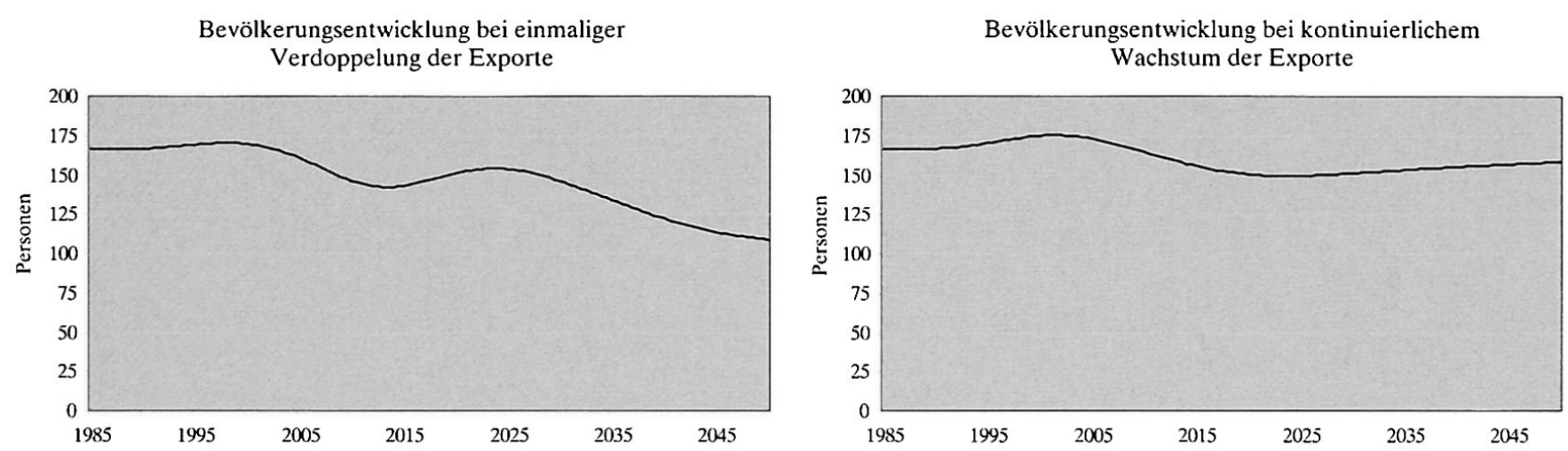

Abbildung 2: Vergleich der Bevölkerungsentwicklung zwischen einem einmaligen (links) und einem kontinuierlichen Wachstum der Exporte (rechts)

Comparison of population development under unique (left) and continuous (right) export growth

Comparaison de l'évolution de la population entre une croissance unique (gauche) et une croissance continue des exportations (droite)

Die Simulationen werden jeweils für einen Zeithorizont von 50 Jahren gerechnet. Dabei geht es nicht darum, genaue Prognosen für einen bestimmten Zeitpunkt in der Zukunft zu erstellen, sondern vielmehr darum, Aussagen über Systemverhalten und -stabilität zu erhalten. Die Dauer des Zeithorizontes ergibt sich aus dem Umstand, dass eine Gemeinde ein komplexes sozioökonomisches System ist, das teilweise lange Anpassungszeiten aufweist (z.B. Altersaufbau der Bevölkerung). Eine lange Simulationsdauer ist daher notwendig, um verzögerte Reaktionen und langfristige Wirkungen von politischen Massnahmen oder veränderten Rahmenbedingungen erfassen zu können.

Eine lange Simulationsdauer führt jedoch auch zu Problemen bei der Quantifizierung des Modells, da es sehr schwierig ist, alle Parameterwerte für einen Zeitraum von 50 Jahren zu schätzen. Im Gegensatz zur InputOutput-Analyse ist es beispielsweise möglich, dass die lokale Wirtschaft Strukturveränderungen erfährt. Damit müssen allerdings Abstriche bei der Genauigkeit der einzelnen Parameterwerte gemacht werden.

\section{Ergebnisse}

Der Artikel von BuSER et al. in diesem Heft beschreibt die Bedeutung von Exportaktivitäten für das regionale Wirtschaftswachstum. In diesem Kapitel wird eine exportorientierte Entwicklungsstrategie aus einer dynamischen Perspektive analysiert. Dabei werden die kurz- und langfristigen Wirkungen einer solchen Strategie auf die regionale Beschäftigung und Bevölkerung untersucht.

Empirische Untersuchungen haben gezeigt, dass die lokale Wirtschaft peripherer und bevölkerungsarmer
Gebiete dann wachsen kann, wenn ihre Güter und Dienstleistungen auf den externen Märkten nachgefragt werden (BUSER 2005 für den Fall der Schweiz; RoBerTs \& THOMSON 2003 für eine internationale Erhebung). Ausmass und Potenzial der externen Nachfrage hängen dabei von verschiedenen Faktoren $a b$. In den folgenden Abschnitten werden die Auswirkungen von möglichen regionalpolitischen Strategien auf die Erschliessung der externen Nachfrage untersucht. Daneben spielen aber die allgemeinen wirtschaftlichen, sozialen und politischen Entwicklungen eine besonders bedeutende Rolle. Dies betrifft beispielsweise die Kaufkraft der Schweizer Bevölkerung, die Präferenzen für Regionalprodukte, die Öffnung internationaler Exportmärkte und die internationale touristische Nachfrage in Abhängigkeit vom Wechselkurs.

Wir stellen in diesem Artikel nur Ergebnisse für Agrargemeinden dar, da diese bezüglich Besiedlung ein besonders kritischer Gemeindetyp sind. Von den 180 Agrargemeinden in der Schweiz ist bei fast 40 Prozent die Besiedlung gefährdet.

\subsection{Export und dezentrale Besiedlung}

Für die Erschliessung der externen Nachfrage haben die verschiedenen Wirtschaftssektoren unterschiedliche Potenziale. Das Simulationsmodell unterscheidet die aggregierten Sektoren «öffentliche Dienstleistungen», «Landwirtschaft», «Industrie und Gewerbe» sowie "private Dienstleistungen», wobei in den beiden letzteren das grösste Wachstumspotenzial liegt.

Abbildung 2 links zeigt die Entwicklung, wenn die Exporte in diesen beiden Wirtschaftssektoren im Jahr 2005 verdoppelt werden. Dieses Experiment beantwortet zum Beispiel die Frage, wie sich eine Politik 
Industrie und Gewerbe

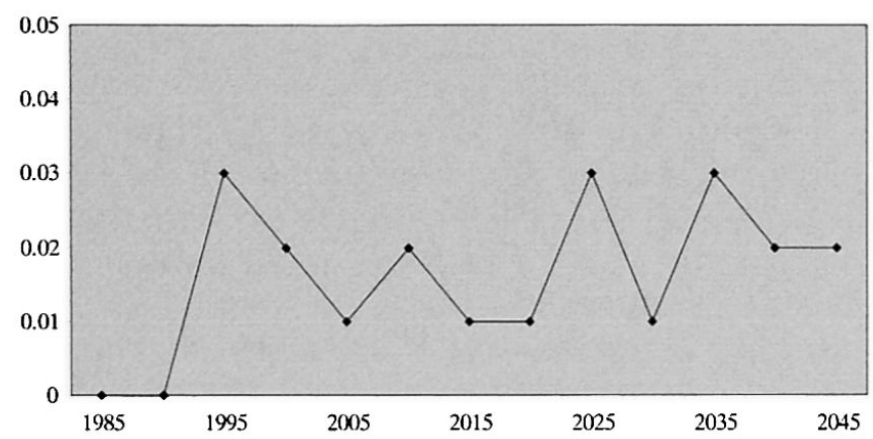

Private Dienstleistungen

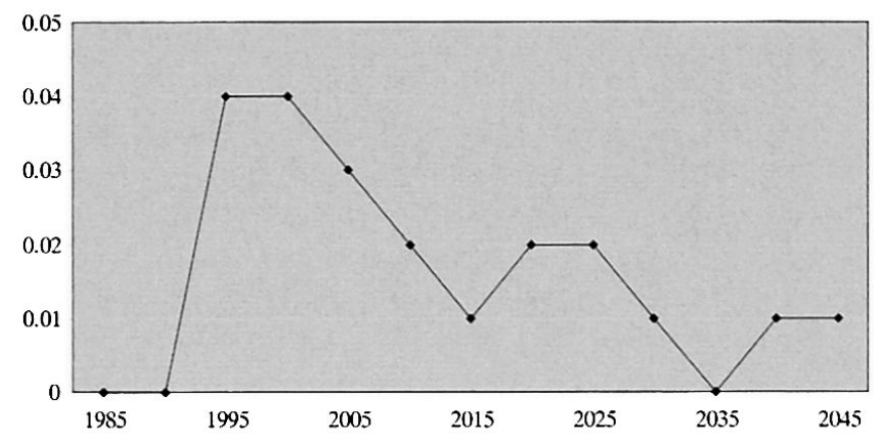

Abbildung 3: Notwendige jährliche Wachstumsrate der Exporte für eine Bevölkerungsstabilisierung

Essential annual export growth rate for population stabilisation

Taux de croissance annuelle des exportations, nécessaire pour une stabilisation démographique

kurz- oder langfristig auswirkt, die auf die Ansiedlung grosser Investitionsfirmen in einer Region abzielt.

Im Vergleich dazu ist rechts eine Situation abgebildet, bei welcher die Exporte im zweiten Sektor sowie in den privaten Dienstleistungen über den gesamten Simulationszeitraum um jährlich $1.5 \%$ wachsen. Eine solche Situation ist dann möglich, wenn die regionale Produktion wettbewerbsfähig erfolgt und die Entwicklung der nationalen und internationalen Märkte zugunsten der Regionalprodukte verläuft.

Der Zusammenhang zwischen Exportaktivitäten und Bevölkerungsentwicklung folgt dabei den in Abbildung 1 beschriebenen Prozessen. Ein Wachstum der Nachfrage führt zu einer Steigerung der Güterproduktion auf dem Produktmarkt. In der Folge werden zusätzliche Arbeitskräfte eingesetzt, die bei entsprechender Attraktivität der Gemeinde durch Einwanderung die Bevölkerungszahl erhöhen. Die daraus entstehende zusätzliche lokale Nachfrage trägt, wenn auch in geringerem Ausmass, zu einer weiteren Verstärkung dieses Prozesses bei. Die Erhöhung der Produktion muss nicht zwingend zu einem Anstieg der Arbeitskräftenachfrage führen. Vielmehr besteht in einer ersten Phase auch die Möglichkeit der Produktivitätssteigerung. Längerfristig wird es aber unumgänglich sein, zusätzliche Arbeitskräfte anzustellen.

Eine Verdoppelung der Exporte ist ein eher unwahrscheinliches Ereignis. Abbildung 2 zeigt aber, dass sogar eine solch extreme Intervention nicht in der Lage ist, die Bevölkerung in Agrargemeinden langfristig zu stabilisieren.

Falls es das Ziel ist, die Bevölkerung langfristig in allen gefährdeten Gemeinden zu stabilisieren, dann haben die Ergebnisse aus Abbildung 2 wichtige politische Implikationen. Sie zeigen, dass ein geringes, jedoch kontinuierliches Wachstum der Exporte wirksamer ist als die Einführung einer umfangreichen wirtschaftlichen Aktivität. Entwicklungsstrategien, die auf die Ansiedlung grosser Investitionsunternehmen abzielen, sollten diese langfristige Perspektive berücksichtigen.

\subsection{Optimaler Export}

In einem nächsten Schritt wird untersucht, wie gross das Wachstum der Exporte sein muss, um die Bevölkerung über längere Zeit hinweg zu stabilisieren. Abbildung 3 zeigt die notwendigen jährlichen Wachstumsraten der Exportaktivitäten in den beiden aggregierten Wirtschaftssektoren «Industrie und Gewerbe» und «private Dienstleistungen» für Agrargemeinden auf. Abbildung 4 vergleicht die aus einer solchen Entwicklung resultierende Beschäftigung in den vier Wirtschaftssektoren mit der Beschäftigungsentwicklung, wenn sich die heutigen Rahmenbedingungen unverändert in die Zukunft fortsetzen (Status quo; bezieht sich insbesondere auf Produktivitätsfortschritte in den verschiedenen Sektoren und das heutige Volumen der Exporte).

Abbildung 3 und 4 zeigen, dass Produktivitätsfortschritte anfänglich ausreichend sind, um die Bevölkerung auf einem konstanten Niveau zu halten. Die Exporte müssen allerdings bereits dann steigen, wenn die Beschäftigungsentwicklung immer noch konstant ist. Ohne diesen Anstieg führen weitere Produktivitätsfortschritte zu einem Beschäftigungsrückgang, wie er im Szenario Status quo beobachtet werden kann. Um eine solche Entwicklung zu verhindern, müssen die Exporte bereits früh ständig an die neueste wirtschaftliche Situation angepasst werden, da die Wirkung auf die Bevölkerung erst mit einer Verzögerung von fünf bis zehn Jahren eintritt. 
Beschäftigungsentwicklung Status quo

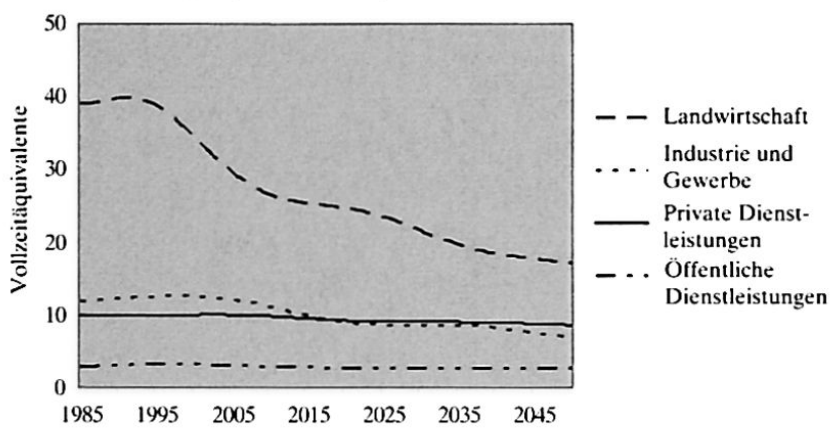

Beschäftigungsentwicklung bei konstanter Bevölkerung

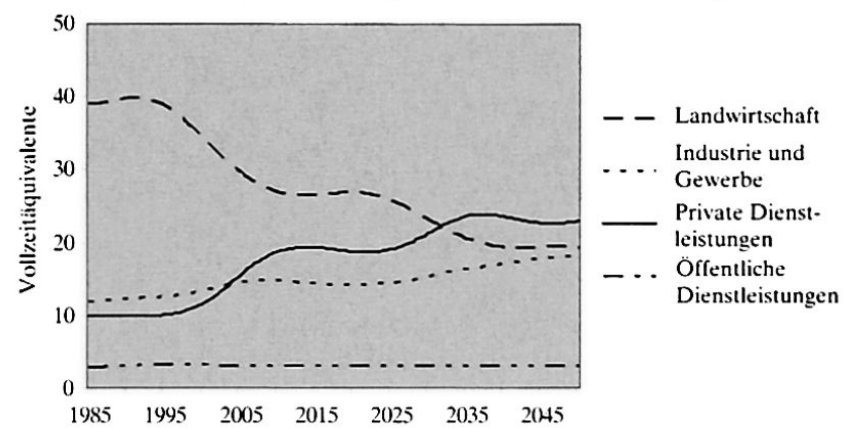

Abbildung 4: Wirtschaftsstruktur im Status quo (links) und für den Fall einer Bevölkerungsstabilisierung (rechts)

Economic structure - current (left) and in the event of population stabilisation (right)

Structure économique de "statu quo" (gauche) et structure dans le cas d'une stabilisation démographique (droite)

Diese Verzögerung stellt eine wichtige Ursache für den Misserfolg von manchen exportorientierten Entwicklungsstrategien dar. Denn oft werden Investitionen in Exportaktivitäten entweder zu früh gestoppt oder erfolgen erst, wenn Beschäftigung und Bevölkerung bereits rückläufig sind. Eine langfristige Sicht bei einer exportorientierten Wachstumsstrategie ist daher zentral.

\subsection{Unternehmertum, Innovation und Export}

Ein Wachstum der Exporte kann auch aus endogen initiierten wirtschaftlichen Aktivitäten entstehen. Lokale und regionale Akteure können die Initiative ergreifen, um dem Beschäftigungsrückgang durch neue wirtschaftliche Aktivitäten Gegensteuer zu geben. Wegen der geringen Grösse der lokalen Märkte müssen diese zu einem grossen Teil auch auf die Exporte ausgerichtet sein. Dies kann durch die Inwertsetzung lokaler Ressourcen in der Landwirtschaft, in Industrie und Gewerbe oder im Tourismus geschehen. Eine weitere Möglichkeit zur Steigerung der Wertschöpfung liegt darin, bisher getätigte Importe durch lokale Produktion zu ersetzen.

Es geht nun darum zu zeigen, unter welchen Bedingungen die externe Nachfrage über lokal initiierte Projekte und Initiativen erschlossen werden kann. Grundsätzlich braucht es dafür verschiedene Akteure - Entscheidungsträger und Beteiligte -, die bereit sind, an der Planung von solchen Projekten teilzunehmen und sich für deren Umsetzung zu engagieren. Erfolgreiche Projekte schlagen sich in der Stabilisierung oder Erhöhung der Beschäftigung und damit der lokalen Bevölkerung nieder und fördern auch die zukünftige Beteiligung an neuen Projekten. Dies wiederum erhöht den Erfolg von Projekten. Dieser Pro- zess kann auch in der entgegengesetzten Richtung verlaufen, so dass sich Beteiligung und Erfolg gegenseitig erodieren und dem Arbeitsplatzverlust kein Gegensteuer gegeben werden kann. Erfolg und Beteiligung können durch das Ausmass der Unterstützung, das die Beteiligten während der Planung und Umsetzung von Projekten erfahren, erhöht werden. Unterstützung bezieht sich im vorliegenden Fall auf die Hilfe durch unternehmerischen Know-how-Transfer, die Bereitstellung von Risikokapital, Netzwerkaufbau, Entlastung im Bereich Administration oder die Bereitstellung von Kommunikationsinfrastruktur. Im Sinne der Neuorientierung der Regionalpolitik erfolgt Unterstützung durch externe Akteure im Rahmen von Public-PrivatePartnerships.

Erfahrungen mit LEADER, einem Programm der EU zur partizipativen Entwicklung und Erprobung integrierter Entwicklungsansätze im ländlichen Raum (SHUcKSMITH 2000), und Untersuchungen zur endogenen Regionalentwicklung in der Schweiz (MühLINGHaus 2002, 2002a) geben Hinweise auf grundlegende Mechanismen und Erfolgsfaktoren lokal initiierter wirtschaftlicher Aktivitäten. Eine Synthese dieser Erfahrungen aus einer Rückkopplungseffekt-orientierten Perspektive ist in Abbildung 5 veranschaulicht.

Abbildung 5 fasst das Spektrum der möglichen Verläufe lokal initiierter wirtschaftlicher Aktivitäten zusammen. Diese Projekte können erfolgreich oder erfolglos sein. Im zeitlichen Verlauf betrachtet ( $\mathrm{x}$-Achse) kann der Anteil erfolgreicher Initiativen (y-Achse) konstant bleiben, steigen oder sinken. Die Ausgangslage in Abbildung 5 ist so, dass nach einer anfänglichen Phase des Gleichgewichts weniger Projekte Erfolge verbuchen können, weil die Zahl der neu lancierten 


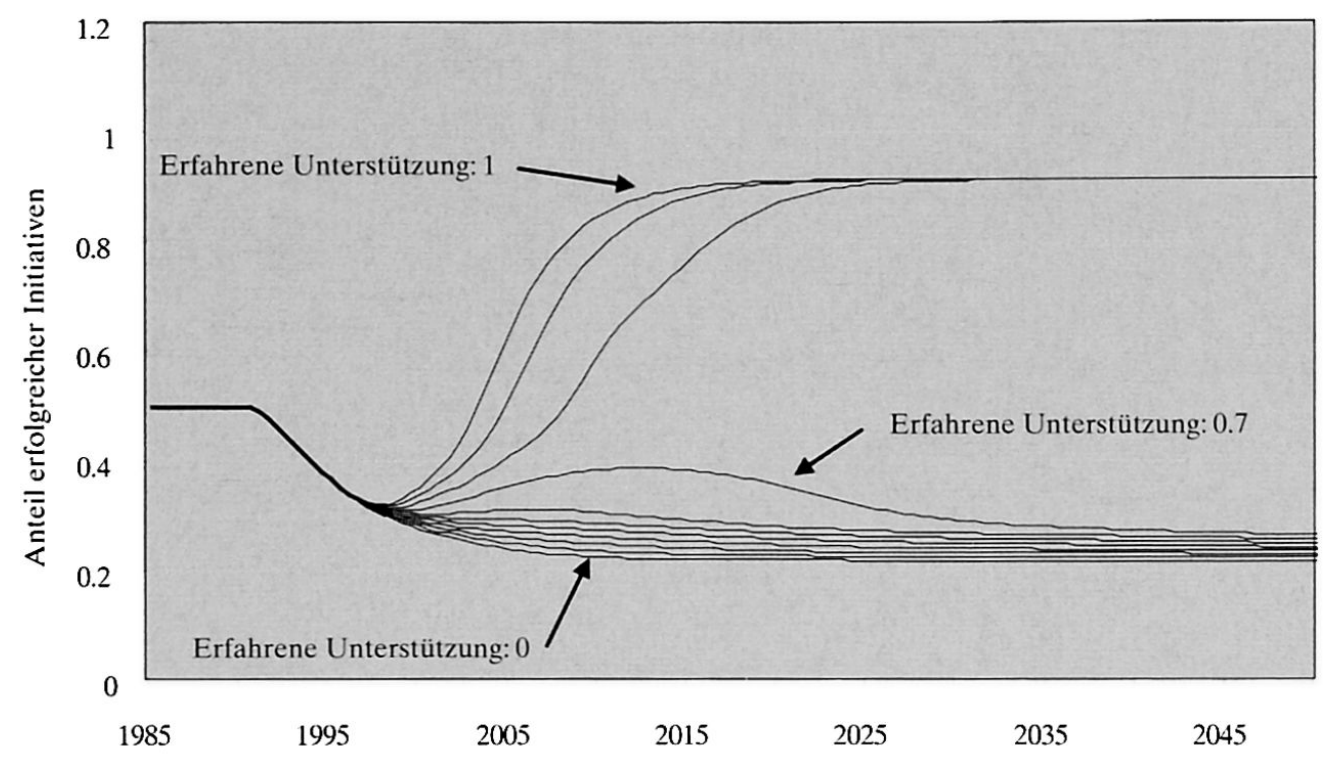

Abbildung 5: Entwicklung des Anteils erfolgreicher Initiativen in Abhängigkeit von der erfahrenen Unterstützung Development of percentage of successful initiatives based on past support

Evolution de la part des initiatives à succès induites par l'expérience

Projektideen zurückgeht und in der Folge auch das Engagement für diese Projekte abnimmt. Um diese Abwärtsbewegung zu stoppen, sind Interventionen nötig, die den vorhandenen Projekten zum Erfolg verhelfen und damit auch die Bereitschaft zur Beteiligung an weiteren Projekten wieder steigern. Eine solche Intervention besteht in den oben beschriebenen Möglichkeiten der Unterstützung lokaler durch externe Akteure.

In Abbildung 5 wird daher das Ausmass der Unterstützung von 0 bis 1 variiert. Die Variable «erfahrene Unterstützung» ist eine qualitative Grösse, die von 0 bis 1 dimensionslos skaliert wird, wobei 0 «keine Unterstützung» und 1 «volle Unterstützung» bedeutet. Auf der $\mathrm{x}$-Achse ist die Zeit aufgetragen, die y-Achse repräsentiert die Output-Variable (Anteil erfolgreicher Initiativen). Wenn man entlang der $\mathrm{x}$-Achse von links nach rechts liest, zeigt jede Linie, wie der Anteil erfolgreicher Initiativen im Zeitablauf auf die erfahrene Unterstützung reagiert. D.h. jede Linie veranschaulicht einen bestimmten Grad der Ausschöpfung des unternehmerischen Potenzials einer Gemeinde oder Region in Abhängigkeit von der Qualität der Partnerschaft zwischen lokalen und externen Akteuren.

Abbildung 5 illustriert, dass der Erfolg von Initiativen stark von der Unterstützung der beteiligten Akteure abhängt. Nach anfänglichem Gleichgewicht bewegen sich Engagement und Erfolg abwärts. Diese Abwärtsbewegung wird etwas abgeschwächt, sobald die lokalen Akteure Unterstützung von Dritten erfahren.
Wenn die Unterstützung hoch genug ist, wächst das Engagement der bisher an der Initiative Beteiligten so stark, dass erste Erfolge eintreten, was das Engagement weiter anwachsen lässt. An diesem Punkt ändert der sich selbst verstärkende Prozess seine Richtung. Ist dieser Richtungswechsel erst erfolgt, bewirkt der Prozess ein rasches Wachstum im Projekterfolg.

Die zentrale Einsicht aus diesen Simulationen besteht darin, dass Entwicklungsstrategien, die auf lokalen Initiativen beruhen, genügend Engagement schaffen müssen, um diesen Richtungswechsel und damit das Wachstum der Beschäftigung und der Bevölkerung zu bewirken.

\section{Fazit}

Die Modellrechnungen im vorangehenden Kapitel zeigen die wirtschaftliche Entwicklung gefährdeter Gemeinden im Wechselspiel zwischen exogenen Marktkräften und endogenen Aktivitäten zur Kompensierung dieser Kräfte auf. Die Anwendung des gewählten Modellierungsansatzes führt zu einigen Erkenntnissen. Die sich selbst verstärkende Wirkung zwischen Bevölkerung, Produktion und Beschäftigung stellt einen dominanten Rückkopplungseffekt dar. Das grundlegende Problem gefährdeter Gemeinden liegt darin, dass die Kräfte, die beispielsweise zu einer Umkehr der Wirkungsrichtung dieses Rückkopplungseffekts führen, sehr gross sein müssen. Die effektivste Entwicklungsstrategie für gefährdete 
Gemeinden liegt in der Exportförderung (vgl. auch Buser et al. in diesem Heft). Aus der Analyse der Rückkoppelungsbeziehungen mit dem dynamischen Simulationsmodell können drei Ursachen für ein langfristiges Scheitern dieser Strategie in der langen Frist identifiziert werden.

Erstens muss bei Investitionen in Exportaktivitäten berücksichtigt werden, dass ein kontinuierliches Wachstum der Exporte effektiver ist als eine einmalige grosse Exportsteigerung. Zweitens ist eine langfristige Sicht bei Investitionen zentral. Eine kurzfristig stabile Beschäftigungsentwicklung bedeutet nicht zwingend, dass die Beschäftigung auch über einen längeren Zeithorizont stabil bleibt, da die Bevölkerungszahl mit grossen zeitlichen Verzögerungen auf die wirtschaftlichen Entwicklungen reagiert. Es ist daher wichtig, Investitionen in Exportaktivitäten nicht zu früh abzubrechen. Drittens kann ein Wachstum bei den Exporten auch aus endogen initiierten wirtschaftlichen Aktivitäten generiert werden. Ein positiver und ein negativer Rückkopplungseffekt bestimmen die Entwicklung von Unternehmertum. Der entscheidende Erfolgsfaktor einer solchen Entwicklungsstrategie beruht darin, dass der positive Rückkopplungseffekt seine Wirkungsrichtung ändert und die Entwicklungsanstrengungen unterstützt. Eine wichtige Ursache für das Scheitern einer solchen Strategie kann sein, dass nicht genügend in Unternehmertum sowie strategische und operative Führungsqualifikationen investiert wird.

Eine erfolgreiche Umsetzung einer exportorientierten Strategie hat weitreichende sozioökonomische Auswirkungen. Typische Agrargemeinden machen beispielsweise eine Transformation hin zu Gemeinden mit starkem zweitem Sektor, zu Wohngemeinden oder zu tourismusorientierten Gemeinden durch.

Die Ergebnisse zeigen, dass die Effektivität der Neuen Regionalpolitik für gefährdete Gemeinden von zwei kritischen Faktoren abhängt. Entscheidungsträger müssen einerseits sicherstellen, dass der Umfang der endogen generierten Exportaktivitäten genügend gross ist. Andererseits brauchen endogen initiierte Projekte externe Unterstützung über einen ausreichend langen Zeitraum hinweg. Die Analysen zeigen aber auch, dass eine Stabilisierung der Bevölkerungszahl in allen gefährdeten Gemeinden nur über ein sehr hohes Wachstum der Exporte oder über beträchtliche öffentliche Transferzahlungen möglich ist. Gemeinden in Pendlerdistanz von regionalen Zentren müssen daher ihre lokalen wirtschaftlichen Aktivitäten mit der Erhöhung der Attraktivität der Gemeinde für Zuzüger verbinden. In jedem Fall müssen die Entwicklungsstrategien für gefährdete Gemeinden und die von regionalen Zentren miteinander kombiniert werden.

\section{Literatur}

ABEGG, C. (2005): Liberalisierung von Netzsektoren: Auswirkungen auf die Unternehmen im Schweizer Alpenraum. - Zürich: vdf Hochschulverlag AG an der ETH Zürich.

Abrahamson, E. \& L. Rosenkopf (1997): Social network effects on the extent of innovation diffusion: a computer simulation. - In: Organization science 8, 3: 289-309.

Armstrong, H. \& J. TAYlor (2000): Regional economics and policy. $-3^{\text {rd }}$ edition, Oxford: Blackwell Publishers.

Buchli, S., Kopainsky, B. \& P. Rieder (2005): Landwirtschaft und dezentrale Besiedlung. - In: Agrarforschung 12, 7: 288-293.

Buser, B. (2005): Regionale Wirtschaftskreisläufe und regionale Wachstumspolitik. Regionalpolitische Prioritäten für unterschiedliche Regionen im Schweizer Alpenraum auf der Basis regionaler Input-Output Tabellen. - Aachen: Shaker Verlag.

Coyle, R.G. (1996): System dynamics modelling. A practical approach. - London: Chapman \& Hall.

EIDGENÖSSISCHES VOLKSWIRTSCHAFTSDEPARTEMENT,EVD (2004): Neue Regionalpolitik. - Bern.

FluRY, C. (2002): Zukunftsfähige Landwirtschaft im Alpenraum. Entwicklung von Nutzungsstrategien für den Kanton Graubünden auf der Basis eines Sektormodells. - Kiel: Wissenschaftsverlag Vauk.

FORRESTER, J.W. (1961): Industrial dynamics. - Waltham, Mass.: Pegasus Communications.

KopaINSKY, B. (2005): A system dynamics analysis of socio-economic development in lagging Swiss regions. - Aachen: Shaker Verlag.

KüPFER, I. (2000): Die regionalwirtschaftliche Bedeutung des Nationalparktourismus: untersucht am Beispiel des schweizerischen Nationalparks. - Zernez: Forschungskommission des Schweizerischen Nationalparks.

MüHLINGHaus, S. (2002): Eigenständige Regionalentwicklung im Schweizer Berggebiet - Umsetzungsprozesse, Erfolgsaussichten und Förderansätze.-St. Gallen: Ostschweizerische Geographische Gesellschaft.

MüHLINGHaus, S. (2002a): Eigenständige Regionalentwicklung als Strategie für periphere ländliche Räume? Eine Untersuchung aus dem Schweizer Berggebiet. In: Geographica Helvetica 57, 2: 127-134.

REPENNING, N. (2002): A simulation-based approach to understanding the dynamics of innovation implementation. - In: Organization science 13, 2: 109-127.

RichaRdSON, G.P. (1991): System dynamics: simulation for policy analysis from a feedback perspective. - In: Fishwick, P.A. \& P.A. LuKer (Hrsg.): Qualitative simulation modeling and analysis. - New York: Springer: 144-169.

RichaRdSON, G.P. \& A.L. PUGH III (1999): Introduction to system dynamics modeling. $-2^{\text {nd }}$ edition, Waltham, Mass.: Pegasus Communications. 
RoberTs, D. \& K.J. Thomson (2003): Sources of structural change in peripheral rural areas: the case of the Western Isles, 1988/89 to 1997. - In: Regional studies 37, 1:61-70.

Ruth, M. \& B. Hannon (1997): Modeling dynamic economic systems. - New York: Springer.

ScHmid, A. (2004): UNESCO Biosphäre Entlebuch: Modell für eine nachhaltige Regionalentwicklung? Konzept Zielerreichungskontrolle. - Schüpfheim: Biosphärenmanagement UNESCO Biosphäre Entlebuch. SCHmid, A., Ruoss, E. \& H. Elsasser (2004): UNESCO Biosphäre Entlebuch: Modell für eine nachhaltige Regionalentwicklung? Konzept Zielerreichungskontrolle. - In: Geographica Helvetica 59, 2: 144-153.

Shucкsmith, M. (2000): Endogenous development, social capital and social inclusion: perspectives from LEADER in the UK. - In: Sociologia ruralis 40, 2: 208-218.

Stalder, U. (2001): Regionale strategische Netzwerke als lernende Organisationen: Regionalförderung aus Sicht der Theorie sozialer Systeme. - = Geographica Bernensia, Reihe G, Grundlagenforschung, 68, Bern: Geographisches Institut der Universität Bern.

STERMAN, J.D. (1988): A skeptic's guide to computer models. - In: Barney, G.O., Kreutzer, W.B. \& M.J. Garrett (Hrsg.): Managing a nation: the microcomputer software catalog. - Boulder, Co.: Westview Press: 209-229.

Sterman, J.D. (2000): Business dynamics. Systems thinking and modeling for a complex world. - Boston: McGraw-Hill.

TerLuin, I.J. (2003): Differences in economic development in rural regions of advanced countries: an overview and critical analysis of theories. - In: Journal of rural studies $19,3: 327-344$.

\section{Zusammenfassung: Wie weiter mit der dezentralen Besiedlung in der Schweiz? Abschätzung von Entwicklungsperspektiven auf Gemeindeebene}

Der vorliegende Artikel beschreibt die Anwendung eines dynamischen Simulationsmodells auf die Frage der Zukunftsperspektiven wirtschaftlich gefährdeter Gemeinden in der Schweiz. Die Modellrechnungen zeigen, dass Beschäftigung und Bevölkerung in gefährdeten Gemeinden insbesondere durch eine Erhöhung der Exporte stabilisiert werden können. Mit dem gewählten Modellansatz können mehrere Ursachen für das Scheitern einer exportorientierten Entwicklungsstrategie identifiziert werden. Eine auf Unternehmertum und Innovation ausgerichtete Regionalpolitik sollte diesen Ursachen Rechnung tragen. Es kann aber auch gezeigt werden, dass eine flächendeckende Erhaltung der dezentralen Besiedlung nur über ein sehr hohes Wachstum der Exporttätigkeit oder durch beträchtliche öffentliche Transferzahlungen möglich ist.
Summary: Where is decentral residential development leading us to in Switzerland? Assessment of developmental possibilities at community level

This article describes the implementation of a dynamic simulation model for investigating possible alternatives faced by communities in Switzerland economically at risk. The model calculations show that employment and population development in risk communities may be stabilised, in particular following export growth. Further, the selected model base allows for the identification of several causes for the failure of export-oriented development strategies. Regional policy directed toward supporting entrepreneurship and innovation should take these causes into account. In addition, it was shown that the trend towards spatially intensive decentral residential development is only viable under a very high growth in export activity or a notable increase in public funding.

Résumé: Quelle suite à donner au peuplement décentralisé en Suisse? Evaluation de perspectives de développement à l'échelle communale

Le présent article décrit l'application d'un modèle de simulation dynamique en réponse à la question des perspectives réservées à des communes suisses économiquement menacées. Les calculs de modélisation montrent que dans les communes menacées, l'emploi et la population peuvent être stabilisés tout particulièrement par une augmentation des exportations. L'approche de modélisation choisie est à même d'identifier plusieurs des causes de l'échec d'une stratégie de développement des exportations. A cet effet, il serait indiqué de promouvoir une politique régionale fondée sur le mouvement entrepreneurial et l'innovation. Il est toutefois également possible de montrer que le maintien d'un peuplement décentralisé sur une aire déterminée n'est possible que par une croissance de très haut niveau des exportations ou par d'imposants transferts financiers publics.

Dr. sc. tech. Birgit Kopainsky, Flury\& Giuliani GmbH, Sonneggstrasse 33, CH-8092 Zürich.

e-mail: birgit.kopainsky@flury-giuliani.ch

Prof. Dr. Peter Rieder, Institut für Agrarwirtschaft, ETH Zentrum, Sonneggstrasse 33, CH-8092 Zürich. e-mail: peter.rieder@iaw.agrl.ethz.ch

\section{Manuskripteingang/received/manuscrit entré le 24.6.2005}

Annahme zum Druck/accepted for publication/accepté pour l'impression: 15.12.2005 